\title{
Defining Carbohydrate Targets on Osteoarthritic Chondrocytes using RT-qPCR and LC-ESI-MS
}

\author{
S.Q. Wu ${ }^{1}$, S. Toegel ${ }^{1}$, M. PABSt ${ }^{2}$, J. Grass ${ }^{2}$, C. ChiARI ${ }^{3}$, \\ F. M. Unger ${ }^{1}$, F. Altmann ${ }^{2}$, H. Viernstein ${ }^{1}$ \\ ${ }^{1}$ Department of Pharmaceutical Technology and Biopharmaceutics, University of Vienna, Vienna, Austria \\ ${ }^{2}$ Department of Chemistry, University of Natural Resources and Applied Life Sciences, Vienna, Austria \\ ${ }^{3}$ Department of Orthopedics, Medical University Vienna, Vienna, Austria \\ E-mail: stefan.toegel@univie.ac.at (S. Toegel)
}

Sci Pharm. 2010; 78: 722

doi:10.3797/scipharm.cespt.8.POT07

Multiple carbohydrate structures in the chondrocyte glycocalyx can serve as targets for biorecognitive lectins. Recently, we have outlined the applicability of selected plant lectins as mediators of bioadhesion in cartilage research [1]. However, given the diversity of glycan structures at cell surfaces as well as the limited specificity of most lectins for certain tissues, in-depth knowledge about altered oligosaccharide structures under disease conditions is required. We therefore aimed at defining in detail the chondrocyte glycome following exposure to pro-inflammatory cytokines.

Primary human chondrocytes were isolated from donors $(n=5)$ and cultured as high density monolayers. Cells were treated with $10 \mathrm{ng} / \mathrm{ml}$ IL-1ß or $40 \mathrm{ng} / \mathrm{ml}$ TNF- $\alpha$. The transcription of 19 glycosyltransferases was quantified using RTqPCR. N- and O-glycan analysis was performed using LC-ESI-MS.

We found that both IL-1ß and TNF- $\alpha$ increased overall sialylation of $\mathrm{N}$ - and $\mathrm{O}$ glycans and induced a major shift from $\alpha 2,6$-linked sialic acid residues towards $\alpha 2,3$-linked sialic acids. These results were supported by RT-qPCR showing increased expression of $\alpha 2,3$ sialyltransferases in treated cells. In this context, ST6Gal1 mRNA levels were markedly reduced 4.7-fold in case of IL-1ß and 3.3fold in case of TNF- $\alpha$, whereas the expression of ST3Gal4 was enhanced 2.8fold and 3.2-fold, respectively. Moreover, we found that both cytokines induced a considerable shift from oligomannosidic glycans towards complex-type Nglycans, whereas core $\alpha 1,6$-fucosylation was found to be reduced particularly by TNF- $\alpha$.

In conclusion, IL-1ß and TNF- $\alpha$ induce a range of specific alterations in chondrocyte glycoproteins, which might be of relevance for malfunctioning cellmatrix interactions in osteoarthritis. Interestingly, such specific changes in the chondrocyte glycome could also be exploited to target functionalized pharmaceutical delivery systems to osteoarthritic chondrocytes.

[1] Toegel S, Harrer N, Plattner VE, Unger FM, Viernstein H, Goldring MB, Gabor F, Wirth M. Lectin binding studies on $\mathrm{C}-28 / \mathrm{I} 2$ and $\mathrm{T} / \mathrm{C}-28 \mathrm{a} 2$ chondrocytes provide a basis for new tissue engineering and drug delivery perspectives in cartilage research. J Control Release 2007; 117: 121-129.

doi:10.1016/j.jconrel.2006.10.004 